\title{
In memoriam Walter Bettschart (1928-2017)
}

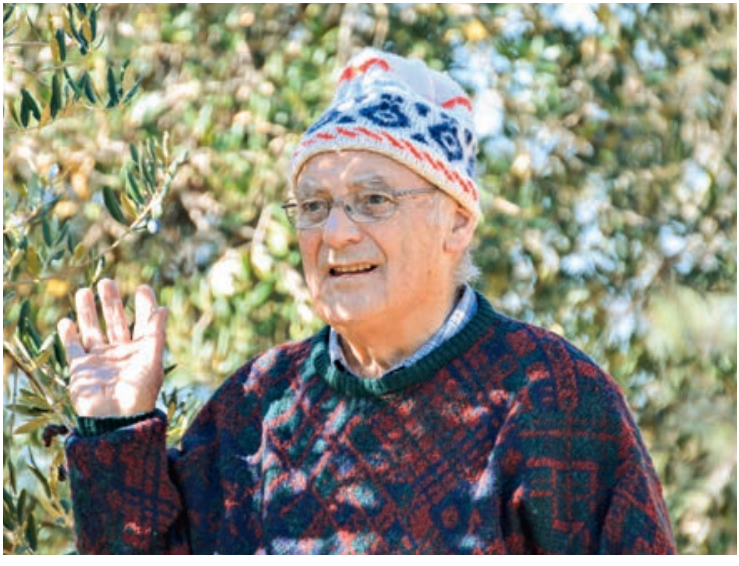

Walter in seinem geliebten Olivenhain in Cotignac (F).

Lieber Walter

schon bald ein dreiviertel Jahr bist du nicht mehr unter uns und doch immer noch präsent: in deiner Familie, bei Deiner Frau, Deinen Kindern und Enkelkindern, aber auch bei vielen Deiner Fach-Kolleginnen und -Kollegen! Da bereits ein Nachruf für Dich im Schweizer Archiv für Neurologie, Psychiatrie und Psychotherapie (Conus, 2018) erschienen ist, erwähne ich hier nur kurz nochmals die «Eckdaten» Deines 90-jährigen Lebens, um dann einige persönliche Erinnerungen an Dich festzuhalten.

Geboren am 16. Januar 1928 in Einsiedeln, warst Du ein Kind deiner Zeit und Deiner Herkunft: aus der bürgerlichen, kultivierten Unternehmer-Familie Bettschart - Dein Vater war Direktor des Benziger Verlages -, deren Schicksal Du in einer eigenen Monographie aufgearbeitet hast (Bettschart, 2015). Wie Deine Brüder durchliefest du das Kollegium der Benediktinermönche in Einsiedeln, mit klassischer Ausrichtung, um Dich dann dem Medizinstudium zu widmen, das Dich von Freiburg i. Ue. über Zürich und Paris bis nach Wien führte. Als junger Arzt betratst Du das Feld der Psychiatrie bzw. der Kinderpsychiatrie, als Du 1958 ins «Institut für Psycho-Hygiene » in Biel eintratst. 1960 begann Deine Karriere im Rahmen des damaligen Office médico-pédagogique, OMP, in Lausanne, das später zum SUPEA [Service universitaire de psychiatrie de l'enfant et de l'adolescent] wurde und dem Du ab 1984 und bis zum Abschluss Deiner beruflichen Karriere 1994 als Direktor und ordentlicher Professor vorstandst.

Doch was alles hast $\mathrm{Du}$ in dieser langen beruflichen Karriere erforscht und geleistet! Psychoanalytisch ausgebildet, warst Du immer am tieferen Sinn und den unbewussten Gründen psychischen Leidens von Kindern und Jugendlichen interessiert. Unentwegt suchtest Du nach neuen und geeigneten Behandlungsmethoden. So wurdest Du zum Pionier des psychoanalytischen Psychodrama für Kinder und Jugendliche, schufst eine der ersten kinderpsychiatrischen Tageskliniken in der Schweiz (Garibian, 2015), sowie ein therapeutisches Behandlungszentrum für Adoleszente und warst stark in der Forschung in unserem Fach engagiert, wofür Du ein eigenes Zentrum aufbauen konntest. Deine fachlichen Qualitäten und Dein grosses Engagement wäre indessen nicht umfassend abgebildet, wenn nicht auch Deine langjährige und intensive Tätigkeit als therapeutischer und institutioneller Supervisor erwähnt würde.

\section{Unentwegt suchtest Du nach neuen und geeigneten Behandlungsmethoden.}

In der Tat hatten wir uns näher kennengelernt, als ich 1992 mit dem Aufbau und der Leitung des KJPD (Kinder- und jugendpsychiatrischer Dienst) in Freiburg i. Ue. betraut wurde. Spontan erklärtest du Dich bereit, mich bei dieser Aufgabe zu unterstützen, wonach ich über mehrere Jahre von Deiner grossen fachlichen und menschlichen Erfahrung profitieren durfte. Danach wurdest du zum psychoanalytisch orientierten Supervisor unserer psychotherapeutischen Équipe, so wie Du auch über viele Jahre die Equipe des «Schwesterdienstes» im Jura supervidiert hast.

Da ich sehr bald den Entschluss fasste, eine kinderpsychiatrische Tagesklinik aufzubauen, also ein Behandlungskonzept umzusetzen, worin Du ja Pionier warst, konnten wir 1994 ein Fachsymposium mit Deiner Präsenz organisieren, das bald darauf zur Gründung einer Stiftung und schliesslich zum Aufbau des CTJ (Centre thérapeutique de jour) in Freiburg führte. 
Im Verlaufe der Jahre sind wir uns näher gekommen, menschlich und fachlich, diskutierten eifrig bei Begegnungen an Kongressen oder Symposien, aber auch anlässlich von freundschaftlichen Treffen in Lausanne und Freiburg - meist verbunden mit einem feinen Essen - und vor zwei Jahren noch anlässlich unseres Besuches bei Dir und Deiner lieben Frau Beryl in Eurer Bastide in Cotignac in Südfrankreich. Deinen geliebten Olivenhain dort hast Du mit Freude und Könnerschaft gepflegt. Im Spätherbst war es dann jeweils schwierig, Daten für die Supervision abzumachen, da die Olivenernte rief!

\section{Wie kein anderer kanntest Du die Geschichte der SGKJPP und unserer europäischen Dach- organisation.}

Nebst Deinem jahrelangen Engagement für die Weiterbildung in unserem Fach - Du hast "gefühlte» drei Revisionen unseres WB-Programmes durchgezogen und Deinem allgemeinen Verdienst für unsere Fachgesellschaft (FG), warst du über Jahrzehnte der Archivar unserer 1957 gegründeten FG, deren 5. Präsident Du warst (1969-1972). Wie kein anderer kanntest Du die Geschichte der SGKJPP sowie auch diejenige der europäischen Dachorganisation unserer Spezialität. In dieser Funktion war es Dir ein grosses Anliegen, dass die sich ablösenden Präsidenten der FG ihre Unterlagen ins Archiv einbrachten. Immer wieder mahntest Du mich, die Ordner aus der Zeit meines Präsidiums (2002-2005) ins Archiv zu bringen und Du schärftest mir ein, dies auch von unsern Nachfolgern zu verlangen.
Unsere letzte Begegnung fand am 19. Nov. vergangen Jahres statt, nur gerade zwei Tage vor Deinem Tod. Ich wollte Dich bei Dir zu Hause besuchen, doch Deine Frau beschied mir, dass Du im Spital seist. So fand ich Dich denn - der Du über all die Jahre mit einer gesunden Rundung in Gesicht und Körper gesegnet warst fast nicht mehr zu erkennen, sichtlich abgemagert. Geistig indessen noch völlig frisch und präsent, begrüsstest Du mich, gemäss unserer bilinguen Sprachpraxis, mit einem fast entschuldigenden: «Patrick, c'est foutu, je vais mourir». Betroffen und schockiert, rief ich Dir in Erinnerung, dass wir doch noch abgemacht hatten, am diesjährigen Festival international de musique sacrée in Fribourg zusammen einen Gregorianik-Workshop zu besuchen. Mit einem milden Augenzwinkern beschiedst du mir: «Gang du nu, i singe dänn mit de Aengel mit»!

Lieber Walter, Du fehlst sehr, nicht nur in Deiner Familie, sondern auch unserem Fach und Deinen Freunden - zu denen zu zählen ich die Freude und das Privileg habe. Adieu!

Patrick Haemmerle

Bibliographie

- Conus P. Walter Bettschart. Médecin, psychiatre et humaniste. Swiss Archives of Neurology, Psychiatry and Psychotherapy. 2018;169(4).

- Bettschart W. Zur Geschichte der Familien Bet(t)schart, besonders der sogenannten Einsiedler Bettschart. Druckerei Franz Kälin AG, Einsiedeln; 2015

- Garibian T. 75 ans de pédopsychiatrie à Lausanne. Du Bercail au Centre psychothérapeutique. Éditions BHMS, Lausanne; 2015. 